Article

\title{
Extensions of Móricz Classes and Convergence of Trigonometric Sine Series in $L^{1}$-Norm
}

\section{Sandeep Kaur Chouhan, Jatinderdeep Kaur * (1D) and Satvinder Singh Bhatia}

Thapar Institute of Engineering and Technology, Patiala, Punjab 147004, India; sandeepchouhan247@gmail.com (S.K.C.); ssbhatia@thapar.edu (S.S.B.)

* Correspondence: jkaur@thapar.edu

Received: 11 September 2018; Accepted: 21 November 2018; Published: 29 November 2018

check for updates

Abstract: In this paper, the extensions of classes $\widetilde{S}, \widetilde{C}$ and $\widetilde{B} V$ are made by defining the classes $\widetilde{S}_{r}, \widetilde{C}_{r}$ and $\widetilde{B} V_{r}, r=0,1,2, \ldots$ It is also shown that class $\widetilde{S}_{r}$ is a subclass of $\widetilde{C}_{r} \cap \widetilde{B} V_{r}$. Moreover, the results on $L^{1}$-convergence of $r$ times differentiated trigonometric sine series have been obtained by considering the $r^{t h}(r=0,1,2, \ldots)$ derivative of modified sine sum under the new extended class $\widetilde{C}_{r} \cap \widetilde{B} V_{r}$.

Keywords: Dirichlet kernel; $L^{1}$-convergence; modified sine sum

\section{Introduction}

Consider the trigonometric sine series

$$
\sum_{k=1}^{\infty} a_{k} \sin k x
$$

where $a_{0}, a_{1}, a_{2}, \ldots$ are the real coefficients. The $n$th partial sum, $S_{n}$, of Series (1) is represented as

$$
S_{n}(x)=\sum_{k=1}^{n} a_{k} \sin k x=-\sum_{k=1}^{n} b_{k}(\cos k x)^{\prime}
$$

where the prime denotes derivatives and $b_{k}=\frac{a_{k}}{k}$. Also, $f(x)=\lim _{n \rightarrow \infty} S_{n}(x)$.

Various conditions are given in the literature (see [1-9]), which guarantee that Series (1) is a Fourier series.

In 1984, Teljakovskii [9] introduced a class $\widetilde{S}$, as follows:

Class $\widetilde{S}$ [9]. A null sequence $\left\{a_{k}\right\}$ is said to belong to class $\widetilde{S}$ if there exists a non-increasing sequence $\left\{B_{k}\right\}$ of numbers s.t.

$$
\begin{gathered}
\left|\Delta b_{k}\right| \leq B_{k} \quad \forall k=1,2,3, \ldots \\
\sum_{k=1}^{\infty} k B_{k}<\infty .
\end{gathered}
$$

where $b_{k}=\frac{a_{k}}{k}, \Delta b_{k}=b_{k}-b_{k+1}$ and proved the following result:

Theorem 1 [9]. If $\left\{a_{k}\right\} \in \widetilde{S}$, then Series (1) is the Fourier series of some function $f \in L^{1}(0, \pi)$.

In 1989, Móricz [5] introduced new classes $\widetilde{B} V$ and $\widetilde{C}$ of the coefficient sequences for the sine series. Class $\widetilde{B} V$ [5]. A null sequence $\left\{a_{k}\right\}$ belongs to $\widetilde{B} V$ if

$$
\sum_{k=1}^{\infty} k\left|\Delta b_{k}\right|<\infty
$$


Class $\widetilde{C}$ [5]. A null sequence $\left\{a_{k}\right\}$ belongs to class $\widetilde{C}$ if for every $\varepsilon>0$ there exists $\delta>0$, independent of $n$, and such that for all $n$,

$$
\int_{0}^{\delta}\left|\sum_{k=n}^{\infty} \Delta b_{k} D_{k}^{\prime}(x)\right| d x \leq \varepsilon .
$$

Here, $D_{k}^{\prime}(x)$ is the first derivative of Dirichlet kernel $\left(D_{k}(x)=\frac{\sin \left(k+\frac{1}{2}\right) x}{2 \sin \frac{x}{2}}\right)$.

Equation (4) implies that, for $1 \leq n \leq N$,

$$
\int_{0}^{\delta}\left|\sum_{k=n}^{N} \Delta b_{k} D_{k}^{\prime}(x)\right| d x \leq 2 \varepsilon .
$$

The following result was proved by Móricz [7].

Theorem 2 [5]. If $\left\{a_{k}\right\} \in \widetilde{B} V$, then

$$
\left\|u_{n}-f\right\| \rightarrow 0 \quad(n \rightarrow \infty) \text { if and only if }\left\{a_{k}\right\} \in \widetilde{C} .
$$

where $u_{n}(x)=S_{n}(x)+b_{n+1} D_{n}^{\prime}(x)$.

The classes $\widetilde{S}, \widetilde{B} V$ and $\widetilde{C}$ seem to be more appropriate for the sine series than the classes $S([7,8])$ $B V$ [10], and $C[3]$ in the ordinary sense. Also, Móricz [5] has proved that $\widetilde{S} \subset \widetilde{B} V \cap \widetilde{C}$.

Motivated by the aforesaid authors, new extended classes $\widetilde{S}_{r}, \widetilde{B} V_{r}$, and $\widetilde{C}_{r}(r=0,1,2, \ldots)$ are defined in this paper as follows:

Class $\widetilde{S}_{r}$. A sequence $\left\{a_{k}\right\}$ is said to belong to class $\widetilde{S}_{r}(r=0,1,2, \ldots)$ if $a_{k} \rightarrow 0$ as $k \rightarrow \infty$, and there exists a non-increasing sequence $\left\{B_{k}\right\}$ of numbers s.t.

$$
\begin{gathered}
\left|\Delta b_{k}\right| \leq B_{k} \quad \forall k=1,2,3, \ldots \\
\sum_{k=1}^{\infty} k^{r+1} B_{k}<\infty, r=0,1,2,3, \ldots
\end{gathered}
$$

where $b_{k}=\frac{a_{k}}{k}, r=0,1,2,3, \ldots$

$$
B_{k} \downarrow 0 \text { and } \sum_{k=1}^{\infty} k^{r+1} B_{k}<\infty \text {, implies that } k^{r+2} B_{k}=o(1) \text { as } k \rightarrow \infty(r=0,1,2, \ldots) .
$$

Remark 1. For $r=0, \widetilde{S}_{r}=\widetilde{S}$.

Remark 2. Obviously, $\widetilde{S}_{r+1} \subset \widetilde{S}_{r}$, but the converse need not be true.

Example 1. Consider a sequence $\Delta b_{n}=\frac{1}{n^{r+3}}, r=0,1,2, \ldots$ and $n \in N$.

$$
a_{n}=n b_{n}=n \sum_{k=n}^{\infty} \Delta b_{k} \leq \sum_{k=n}^{\infty} \frac{k}{k^{r+3}}=\sum_{k=n}^{\infty} \frac{1}{k^{r+2}} \rightarrow 0 \text { as } n \rightarrow \infty .
$$

Choose $B_{n}=\frac{1}{n^{r+3}}, r=0,1,2, \ldots \quad \forall n$. Clearly, $B_{n} \downarrow 0$ as $n \rightarrow \infty$ and $\left|\Delta b_{n}\right| \leq B_{n} \forall n$. Consider the series

$$
\sum_{n=1}^{\infty} n^{r+1} B_{n}=\sum_{n=1}^{\infty} n^{r+1} \frac{1}{n^{r+3}} \approx \sum_{n=1}^{\infty} \frac{1}{n^{2}} \quad \text { which is convergent. }
$$


This implies $\left\{a_{n}\right\} \in \widetilde{S}_{r}$.

But the series $\sum_{n=1}^{\infty} n^{r+2} B_{n} \approx \sum_{n=1}^{\infty} \frac{1}{n}$ is divergent.

This implies that $\left\{a_{n}\right\}$ does not belong to class $\widetilde{S}_{r+1}$.

Class $\widetilde{B} V_{r}$. A null sequence $\left\{a_{k}\right\}$ belongs to $\widetilde{B} V_{r},(r=0,1,2, \ldots)$ if

$$
\sum_{k=1}^{\infty} k^{r+1}\left|\Delta b_{k}\right|<\infty
$$

Remark 3. For $r=0, \widetilde{B} V_{r}=\widetilde{B} V$.

Remark 4. Clearly, $\widetilde{B} V_{r+1} \subset \widetilde{B} V_{r},(r=0,1,2, \ldots)$, but the converse may not be true.

Class $\widetilde{C}_{r}$. A null sequence $\left\{a_{k}\right\}$ belongs to class $\widetilde{C}_{r}(r=0,1,2, \ldots)$, if for every $\varepsilon>0$, there exists $\delta>0$, independent of $n$, and such that for all $n$,

$$
\int_{0}^{\delta}\left|\sum_{k=n}^{\infty} \Delta b_{k} D_{k}^{r+1}(x)\right| d x \leq \varepsilon
$$

Here, $D_{k}^{r+1}(x)$ is the $(r+1)^{t h}$ derivative of Dirichlet kernel.

Equation (4) implies, for $1 \leq n \leq N$,

$$
\int_{0}^{\delta}\left|\sum_{k=n}^{N} \Delta b_{k} D_{k}^{r+1}(x)\right| d x \leq 2 \varepsilon
$$

Remark 5. For $r=0, \widetilde{C}_{r}=\widetilde{C}$.

Remark 6. It is obvious that $\widetilde{\mathrm{C}}_{r+1} \subset \widetilde{\mathrm{C}}_{r}$ but the converse need not be true.

Example 2. Define $\Delta b_{n}=\frac{1}{n^{r+3}}, r=0,1,2, \ldots$ and $n=1,2,3, \ldots$

$$
a_{n}=n b_{n}=n \sum_{k=n}^{\infty} \Delta b_{k} \leq \sum_{k=n}^{\infty} \frac{k}{k^{r+3}}=\sum_{k=n}^{\infty} \frac{1}{k^{r+2}} \rightarrow 0 \text { as } n \rightarrow \infty .
$$

Consider, the integral

$$
\begin{gathered}
\int_{0}^{\pi}\left|\sum_{k=n}^{\infty} \Delta b_{k} D_{k}^{r+2}(x)\right| d x=\sum_{k=n}^{\infty} \frac{1}{n^{r+3}} \int_{0}^{\pi}\left|D_{k}^{r+2}(x)\right| d x=O\left(\sum_{k=n}^{\infty} \frac{1}{n^{r+3}}\left(n^{r+2} \log n\right)\right) \\
=O\left(\sum_{k=n}^{\infty} \frac{\log n}{n}\right)
\end{gathered}
$$

which is divergent.

However,

$$
\begin{gathered}
\int_{0}^{\pi}\left|\sum_{k=n}^{\infty} \Delta b_{k} D_{k}^{r+1}(x)\right| d x=\sum_{k=n}^{\infty} \frac{1}{n^{r+3}} \int_{0}^{\pi}\left|D_{k}^{r+1}(x)\right| d x=O\left(\sum_{k=n}^{\infty} \frac{1}{n^{r+3}}\left(n^{r+1} \log n\right)\right) \\
=O\left(\sum_{k=n}^{\infty} \frac{\log n}{n^{2}}\right) \text { which is convergent. }
\end{gathered}
$$


Therefore $\left\{a_{n}\right\} \in \widetilde{C}_{r}$.

Lemmas related to the main results are given in Section 2. The Section 3 comprises the main results of this paper. Firstly, in this section, we have shown that the new extended class $\widetilde{S}_{r}$ is a subclass of $\widetilde{C}_{r} \cap \widetilde{B} V_{r}(r=0,1,2, \ldots)$. Moreover, the theorems are presented concerning the $L^{1}$ convergence of trigonometric sine series using modified sine sum [11], defined as

$$
\beta_{n}(x)=\sum_{k=1}^{n}\left(\frac{a_{k+1}}{k+1}+\sum_{j=k}^{n} \Delta^{2}\left(\frac{a_{j}}{j}\right)\right) k \sin k x
$$

under the extended classes of numerical sequences.

\section{Lemmas}

Lemma 1. [6] Let $n \geq 1$ and $r$ be a nonnegative integer $x \in[\varepsilon, \pi]$. Then, $\left|D_{n}^{r}(x)\right| \leq \frac{C n^{r}}{x}$, where $C$ denotes a positive absolute constant.

Lemma 2. [6] $\left\|D_{n}^{r}(x)\right\|_{L^{1}}=O\left(n^{r} \log n\right), r=0,1,2, \ldots$ where $D_{n}^{r}(x)$ represents the $r^{\text {th }}$ derivative of the Dirichlet kernel.

\section{Main Results}

Theorem 3. The following relation holds $\widetilde{S}_{r} \subset \widetilde{C}_{r} \cap \widetilde{B} V_{r}$ for each $r \in\{0,1,2, \ldots\}$.

Proof. It is plain that $\widetilde{S}_{r} \subset \widetilde{B} V_{r}$.

In order to prove that $\widetilde{S}_{r} \subset \widetilde{C}_{r}$ we take a sequence $\left\{a_{k}\right\}$ in $\widetilde{S}_{r}$ and consider

$$
\int_{0}^{\pi}\left|\sum_{k=n}^{\infty} \Delta b_{k} D_{k}^{r+1}(x)\right| d x ; \text { where } b_{k}=\frac{a_{k}}{k}
$$

If we apply summation by parts, we obtain

$$
\begin{gathered}
\int_{0}^{\pi}\left|\sum_{k=n}^{\infty} \Delta b_{k} D_{k}^{r+1}(x)\right| d x \\
\leq \lim _{N \rightarrow \infty}\left[\sum_{k=n}^{N-1} \Delta B_{k} \int_{0}^{\pi}\left|\sum_{j=0}^{k} \frac{\Delta b_{j}}{B_{j}} D_{j}^{r+1}(x)\right| d x+B_{N} \int_{0}^{\pi}\left|\sum_{K=0}^{N} \frac{\Delta b_{k}}{B_{k}} D_{k}^{r+1}(x)\right| d x\right. \\
\left.+B_{n} \int_{0}^{\pi}\left|\sum_{K=0}^{n-1} \frac{\Delta b_{k}}{B_{k}} D_{k}^{r+1}(x)\right| d x\right]
\end{gathered}
$$

Clearly $\left|\frac{\Delta b_{k}}{B_{k}}\right| \leq 1$. Now, if we first apply Bernstein's inequality [12] and then Sidon Fomin's inequality $([1,7])$, we get

$$
\begin{aligned}
& \int_{0}^{\pi}\left|\sum_{k=0}^{n} \frac{\Delta b_{k}}{B_{k}} D_{k}^{(r+1)}(x)\right| d x \leq M(n+1)^{r+2}, \quad r=0,1,2, \ldots \\
& \int_{0}^{\pi}\left|\sum_{k=n}^{\infty} \Delta b_{k} D_{k}^{r+1}(x)\right| d x \leq \lim _{N \rightarrow \infty}\left\{\sum_{k=n}^{N-1} \Delta B_{k}(k+1)^{r+2}+B_{N}(N+1)^{r+2}\right\}+n^{r+2} B_{n} \\
& =\sum_{k=n}^{\infty}\left[(k+1)^{r+2}-k^{r+2}\right] B_{k}+n^{r+2} B_{n} \\
& =O\left(\sum_{k=n}^{\infty} k^{r+1} B_{k}\right)+n^{r+2} B_{n}
\end{aligned}
$$


So, by given hypothesis, we have

$$
\int_{0}^{\pi}\left|\sum_{k=n}^{\infty} \Delta b_{k} D_{k}^{r+1}(x)\right| d x \leq \frac{\varepsilon}{2} \text { if } n \text { is large enough say } n \geq n_{0} .
$$

For any $1 \leq n \leq N$, we can estimate as follows:

$$
\begin{gathered}
\int_{0}^{\delta}\left|\sum_{k=n}^{N} \Delta b_{k} D_{k}^{r+1}(x)\right| d x \leq \int_{0}^{\delta}\left|\sum_{k=n}^{n_{0}} \Delta b_{k} D_{k}^{r+1}(x)\right| d x+\int_{0}^{\delta}\left|\sum_{k=n_{0}}^{N} \Delta b_{k} D_{k}^{r+1}(x)\right| d x \\
\leq \frac{1}{2} \delta \sum_{k=1}^{n_{0}} k(k+1)^{r+1}\left|\Delta b_{k}\right|+\frac{\varepsilon}{2}<\varepsilon
\end{gathered}
$$

provided $\delta$ is small enough. This proves that $\left\{a_{k}\right\} \in \widetilde{C}_{r}$.

Theorem 4. Let $\left\{a_{k}\right\}$ be a sequence of numbers belonging to the class $\widetilde{C} \cap \widetilde{B} V$ and if $\lim _{n \rightarrow \infty} a_{n} \log n=0$, then

$$
\left\|\beta_{n}-f\right\|=o(1), n \rightarrow \infty
$$

Proof. The modified trigonometric sine sum is given by

$$
\begin{aligned}
\beta_{n}(x) & =\sum_{k=1}^{n}\left(\frac{a_{k+1}}{k+1}+\sum_{j=k}^{n} \Delta^{2}\left(\frac{a_{j}}{j}\right)\right) k \sin k x \\
= & \sum_{k=1}^{n} a_{k} \sin k x+\left(\frac{a_{n+2}}{n+2}-\frac{a_{n+1}}{n+1}\right) \sum_{k=1}^{n} k \sin k x \\
= & -\sum_{k=1}^{n} b_{k}(\cos k x)^{\prime}-\left(b_{n+2}-b_{n+1}\right) D_{n}^{\prime}(x)
\end{aligned}
$$

By using the summation by parts, we get

$$
\beta_{n}=-\sum_{k=1}^{n} \Delta b_{k} D_{k}^{\prime}(x)-b_{n} D_{n}^{\prime}(x)-\left(b_{n+2}-b_{n+1}\right) D_{n}^{\prime}(x)
$$

Under the given hypothesis and Lemma 1 , series $\sum_{k=1}^{n} \Delta b_{k} D_{k}^{\prime}(x)$ converges absolutely and $b_{n} D_{n}^{\prime}(x) \rightarrow 0$ as $n \rightarrow \infty$.

Hence $\lim _{n \rightarrow \infty} \beta_{n}(x)=f(x)$ exists in $(0, \pi)$.

Next, consider

$$
\begin{aligned}
\left\|f(x)-\beta_{n}(x)\right\| & =\left\|\sum_{k=n+1}^{\infty} a_{k} \sin k x-\left(\frac{a_{n+2}}{n+2}-\frac{a_{n+1}}{n+1}\right) \sum_{k=1}^{n} k \sin k x\right\| \\
& =\int_{0}^{\pi}\left|-\sum_{k=n+1}^{\infty} b_{k}(\cos k x)^{\prime}-\left(b_{n+1}-b_{n+2}\right) D_{n}^{\prime}(x)\right| d x
\end{aligned}
$$

By using Abel's transformation, we have

$$
\begin{aligned}
& =\int_{0}^{\pi}\left|-\sum_{k=n+1}^{\infty} \Delta b_{k} D_{k}^{\prime}(x)+b_{n+2} D_{n}^{\prime}(x)\right| d x \\
& =\int_{0}^{\pi}\left|\sum_{k=n+1}^{\infty} \Delta b_{k} D_{k}^{\prime}(x)\right| d x+\frac{n}{n+2} a_{n+2} \log n
\end{aligned}
$$


The second term of the above equation is of $o(1)$ as $a_{n} \log n=0$ as $n \rightarrow \infty$. For the remaining part, let $\varepsilon>0$, then there exists $\delta>0$, such that

$$
\int_{0}^{\delta}\left|\sum_{k=n+1}^{\infty} \Delta b_{k} D_{k}^{\prime}(x)\right| d x<\frac{\varepsilon}{2} \text { for all } n \geq 0 .
$$

Then

$$
\begin{aligned}
\int_{0}^{\pi}\left|\sum_{k=n+1}^{\infty} \Delta b_{k} D_{k}^{\prime}(x)\right| d x=\int_{0}^{\delta} \mid \sum_{k=n+1}^{\infty} & \Delta b_{k} D_{k}^{\prime}(x)\left|d x+\int_{\delta}^{\pi}\right| \sum_{k=n+1}^{\infty} \Delta b_{k} D_{k}^{\prime}(x) \mid d x \\
& \leq \frac{\epsilon}{2}+\sum_{k=n+1}^{\infty}\left|\Delta b_{k}\right| \int_{\delta}^{\pi}\left|D_{k}^{\prime}(x)\right| d x \\
& \leq \frac{\epsilon}{2}+C \sum_{k=n+1}^{\infty} k\left|\Delta b_{k}\right| \int_{\delta}^{\pi} d x / x^{2} \\
& \leq \frac{\epsilon}{2}+C \delta^{-1} \sum_{k=n+1}^{\infty} k\left|\Delta b_{k}\right| \leq \varepsilon
\end{aligned}
$$

This proves that $\left\|f(x)-\beta_{n}(x)\right\|=o(1)$ as $n \rightarrow \infty$.

Theorem 5. Let $\left\{a_{k}\right\}$ be a sequence of numbers belonging to the class $\widetilde{C} \cap \widetilde{B} V$, and if $\lim _{n \rightarrow \infty} a_{n} \log n=0$, then

$$
\left\|S_{n}-f\right\|=o(1), \quad n \rightarrow \infty .
$$

Proof. $\left\|S_{n}-f\right\| \leq\left\|S_{n}-\beta_{n}\right\|+\left\|\beta_{n}-f\right\|$

$$
\begin{gathered}
\leq\left|b_{n+1}\right| \int_{0}^{\pi}\left|D_{n}^{\prime}(x)\right| d x+\left|b_{n+2}\right| \int_{0}^{\pi}\left|D_{n}^{\prime}(x)\right| d x+o(1) \\
\leq\left|a_{n+1}\right| \log n+\left|a_{n+2}\right| \log n(b y \text { Lemma } 2) \\
=o(1), n \rightarrow \infty
\end{gathered}
$$

Theorem 6. Let $\left\{a_{k}\right\}$ be a sequence of numbers belonging to the class $\widetilde{C}_{r} \cap \widetilde{B} V_{r}$ and if $n^{r} a_{n} \log n=$ 0 , as $n \infty$, for each $r=0,1,2, \ldots$ Then

$$
\left\|\beta_{n}^{r}(x)-f^{r}(x)\right\|=o(1), \quad n \rightarrow \infty
$$

Here, $f^{r}(x)$ is the rth derivative of $f(x)$, where $r=0,1,2, \ldots$

Proof. Consider the modified trigonometric sine sum as

$$
\begin{aligned}
\beta_{n}(x) & =\sum_{k=1}^{n}\left(\frac{a_{k+1}}{k+1}+\sum_{j=k}^{n} \Delta^{2}\left(\frac{a_{j}}{j}\right)\right) k \sin k x \\
= & \sum_{k=1}^{n} a_{k} \sin k x+\left(\frac{a_{n+2}}{n+2}-\frac{a_{n+1}}{n+1}\right) \sum_{k=1}^{n} k \sin k x
\end{aligned}
$$

Taking $r$-times differentiation of $\beta_{n}(x)$, we get 


$$
\begin{gathered}
\beta_{n}^{r}(x)=S_{n}^{r}(x)+\left(\frac{a_{n+2}}{n+2}-\frac{a_{n+1}}{n+1}\right) \sum_{k=1}^{n} k^{r+1} \sin \left(k x+\frac{r \pi}{2}\right) \\
=\sum_{k=1}^{n} k^{r} a_{k} \sin \left(k x+\frac{r \pi}{2}\right)+\left(\frac{a_{n+1}}{n+1}-\frac{a_{n+2}}{n+2}\right) \sum_{k=1}^{n} k^{r+1} \cos \left(k x+\frac{(r+1) \pi}{2}\right) \\
=-\sum_{k=1}^{n} k^{r+1} b_{k} \cos \left(k x+\frac{(r+1) \pi}{2}\right)+\left(b_{n+1}-b_{n+2}\right) D_{n}^{r+1}(x)
\end{gathered}
$$

If we apply Abel's transformation on the first term of above equation, we get

$$
\begin{gathered}
\beta_{n}^{r}(x)=-\sum_{k=1}^{n-1} \Delta b_{k} D_{k}^{r+1}(x)-b_{n} D_{n}^{r+1}(x)+\left(b_{n+1}-b_{n+2}\right) D_{n}^{r+1}(x) \\
=-\sum_{k=1}^{n} \Delta b_{k} D_{k}^{r+1}(x)-b_{n+2} D_{n}^{r+1}(x)
\end{gathered}
$$

The series $\sum_{k=1}^{\infty} \Delta b_{k} D_{k}^{r+1}(x)$ converges absolutely and $b_{n} D_{n}^{r+1}(x) \rightarrow 0$ as $n \rightarrow \infty$ using Lemma 1 and given hypothesis.

Therefore $\lim _{n \rightarrow \infty} \beta_{n}^{r}(x)=f^{r}(x)$ exists in $(0, \pi)$.

Next, consider

$$
\begin{gathered}
\left\|f(x)-\beta_{n}(x)\right\|=\left\|\sum_{k=n+1}^{\infty} a_{k} \sin k x-\left(\frac{a_{n+2}}{n+2}-\frac{a_{n+1}}{n+1}\right) \sum_{k=1}^{n} k \sin k x\right\| \\
\left\|f^{r}(x)-\beta_{n}^{r}(x)\right\| \quad=\| \sum_{k=n+1}^{\infty} k^{r} a_{k} \sin \left(k x+\frac{r \pi}{2}\right) \\
-\left(\frac{a_{n+2}}{n+2}-\frac{a_{n+1}}{n+1}\right) \sum_{k=1}^{n} k^{r+1} \sin \left(k x+\frac{r \pi}{2}\right) \| \\
=\left\|\sum_{k=n+1}^{\infty} k^{r} a_{k} \sin \left(k x+\frac{r \pi}{2}\right)+\left(\frac{a_{n+2}}{n+2}-\frac{a_{n+1}}{n+1}\right) \sum_{k=1}^{n} k^{r+1} \cos \left(k x+\frac{(r+1) \pi}{2}\right)\right\| \\
=\int_{0}^{\pi}\left|-\sum_{k=n+1}^{\infty} k^{r+1} b_{k} \cos \left(k x+\frac{(r+1) \pi}{2}\right)+\left(b_{n+2}-b_{n+1}\right) D_{n}^{r+1}(x)\right| d x
\end{gathered}
$$

If we apply Abel's transformation, we obtain

$$
\begin{gathered}
=\int_{0}^{\pi}\left|-\sum_{k=n+1}^{\infty} \Delta b_{k} D_{k}^{r+1}(x)+b_{n+1} D_{n}^{r+1}(x)-b_{n+1} D_{n}^{r+1}(x)+b_{n+2} D_{n}^{r+1}(x)\right| d x \\
\leq \int_{0}^{\pi}\left|\sum_{k=n+1}^{\infty} \Delta b_{k} D_{k}^{r+1}(x)\right| d x+\left|b_{n+2}\right| \int_{0}^{\pi}\left|D_{n}^{r+1}(x)\right| d x \\
\leq \int_{0}^{\pi}\left|\sum_{k=n+1}^{\infty} \Delta b_{k} D_{k}^{r+1}(x)\right| d x+\frac{a_{n+2}}{n+2} n^{r+1} \log n
\end{gathered}
$$

The second term of the above equation are of $o(1)$ as $n^{r} a_{n} \log n=0$ as $n \rightarrow \infty$. For the remaining part, let $\varepsilon>0$, then there exists $\delta>0$, such that $\int_{0}^{\delta}\left|\sum_{k=n+1}^{\infty} \Delta b_{k} D_{k}^{r+1}(x)\right| d x<\varepsilon / 2$ for all $n \geq 0$. Then

$$
\begin{aligned}
\int_{0}^{\pi}\left|\sum_{k=n+1}^{\infty} \Delta b_{k} D_{k}^{r+1}(x)\right| d x= & \int_{0}^{\delta}\left|\sum_{k=n+1}^{\infty} \Delta b_{k} D_{k}^{r+1}(x)\right| d x+\int_{\delta}^{\pi}\left|\sum_{k=n+1}^{\infty} \Delta b_{k} D_{k}^{r+1}(x)\right| d x \\
& \leq \frac{\varepsilon}{2}+\sum_{k=n+1}^{\infty}\left|\Delta b_{k}\right| \int_{\delta}^{\pi}\left|D_{k}^{r+1}(x)\right| d x \\
\leq & \leq \frac{\varepsilon}{2}+C \sum_{k=n+1}^{\infty} k^{r+1}\left|\Delta b_{k}\right| \int_{\delta}^{\pi} d x / x^{r+2} \\
\leq \frac{\varepsilon}{2}+C \delta^{-(r+1)} & \sum_{k=n+1}^{\infty} k^{r+1}\left|\Delta b_{k}\right| \leq \varepsilon \quad \text { (by given hypothesis) }
\end{aligned}
$$

Therefore, $\left\|f^{r}(x)-\beta_{n}^{r}(x)\right\|_{L^{1}}=o(1)$ as $n \rightarrow \infty$. 
Remark 7. For $r=0$, Theorem 6 reduces to Theorem 4.

Theorem 7. Let $\left\{a_{k}\right\}$ be a sequence of numbers belonging to the class $\widetilde{C}_{r} \cap \widetilde{B} V_{r}$ and if $n^{r} a_{n} \log n=o(1)$ as $n \rightarrow \infty$. Then

$$
\left\|S_{n}^{r}(x)-f^{r}(x)\right\|=o(1), n \rightarrow \infty
$$

where $r=0,1,2 \ldots$.

Proof. $\left\|S_{n}^{r}-f^{r}\right\| \leq\left\|S_{n}^{r}-\beta_{n}^{r}\right\|+\left\|\beta_{n}^{r}-f^{r}\right\|$

$$
\begin{gathered}
\leq\left|b_{n+2}\right| \int_{0}^{\pi}\left|D_{n}^{r+1}(x)\right| d x+\left|b_{n+1}\right| \int_{0}^{\pi}\left|D_{n}^{r+1}(x)\right| d x+o(1) \\
\leq \frac{\left|a_{n+2}\right|}{n+2} n^{r+1} \log n+\frac{\left|a_{n+1}\right|}{n+1} n^{r+1} \log n \\
=o(1) \text { as } n \rightarrow \infty
\end{gathered}
$$

Remark 8. For $r=0$, Theorem 7 reduces to Theorem 5 .

Remark 9. Combining Theorem 6 and Theorem 7 with Theorem 3, the following result holds:

Corollary 1. If $\left\{a_{k}\right\} \in \widetilde{S}_{r}(r=0,1,2,3, \ldots)$ and if $n^{r} a_{n} \log n=o(1)$ as $n \rightarrow \infty$. Then

(i) $\left\|\beta_{n}^{r}(x)-f^{r}(x)\right\|=o(1), n \rightarrow \infty$.

(ii) $\left\|S_{n}^{r}(x)-f^{r}(x)\right\|=o(1), n \rightarrow \infty$.

Author Contributions: All authors have contributed in obtaining the new results presented in this article. All authors read and approved the final manuscript. Investigation, S.K.C.; Supervision, J. K. and S.S.B.

Funding: This research received no external funding.

Conflicts of Interest: The authors declare that they have no conflicts of interest.

\section{References}

1. Fomin, G.A. On linear method for summing Fourier series. Mat. Sb (Russ.) 1964, 64, 144-152.

2. Fomin, G.A. A class of trigonometric series. Math. Notes 1978, 23, 117-123. [CrossRef]

3. Garrett, J.W.; Stanojevic, C.V. Necessary and Sufficient conditions for $L^{1}$-convergence of trigonometric series. Proc. Am. Math. Soc. 1976, 60, 68-71.

4. Kano, T. Coefficients of some trigonometric series. J. Fac. Sci. Shinshu Univ. 1968, 3, 153-162.

5. Móricz, F. On the integrability and $L^{1}$-convergence of sine series. Studia Math. 1989, 335, 187-200. [CrossRef]

6. Sheng, S.Y. The extension of the theorems of Č.V. Stanojevic and V.B. Stanojevic. Proc. Am. Math. Soc. 1990, 110, 895-904. [CrossRef]

7. Sidon, S. Hinreichende Bedingungen fur den Fourier-charakter einer trigonometrischen Reihe. J. Lond. Math. Soc. 1939, 14, 158-160. [CrossRef]

8. Telyakovskii, S.A. On a sufficient condition of Sidon for integrability of trigonometric series. Mat. Zametki 1973, 14, 317-328. [CrossRef]

9. Telyakovskii, S.A. On the integrability of sine series. Trudy Mat. Inst. Steklov. 1984, 163, 229-233.

10. Bary, N.K. A Treatise on Trigonometric Series; Pergamon Press: London, UK, 1964; Volumes I, II.

11. Chouhan, S.K.; Kaur, J.; Bhatia, S.S. Convergence and Summability of Fourier sine and cosine series with its applications. Proc. Natl. Acad. Sci. India Sect. A Phys. Sci. 2018, 1-8. [CrossRef]

12. Zygmund, A. Trigonometric Series; Cambridge University Press: Cambridge, UK, 1959.

(C) 2018 by the authors. Licensee MDPI, Basel, Switzerland. This article is an open access article distributed under the terms and conditions of the Creative Commons Attribution (CC BY) license (http:/ / creativecommons.org/licenses/by/4.0/). 